\title{
THE SOCIAL CONTEXT OF CONTACT CALLS BY ROOKS (CORVUS FRUGILEGUS)
}

\author{
Alexandru MUNTEANU*, DVM \\ Professor lonel PAPUC*, PhD \\ Ira G. FEDERSPIEL **, PhD \\ Professor Nicola S. CLAYTON ${ }^{\star \star \star}$, PhD \\ Nathan J. EMERY**, MD
}

\begin{abstract}
Communication is the link between individuals of one species and represents the essence of social life. Vocal communication is one of the most studied forms of information exchange, although it also comes with interspecific barriers that are still tricky to overcome. While we are able to understand the meaning of another human's words, we fail to understand an animal's utterances. Among these, bird song has become a field of particular interest. However, little is known yet about many species' vocalizations, and even less about their significance or how different factors influence them. The presented study establishes the vocal repertoire of a group of rooks and further investigates the importance of contact calls between partners in an experiment. We found that test subjects and other group members produced more contact calls after than before partners had been separated from each other, indicating stress induced by physical isolation and/or the lack of visual contact as an important factor influencing the call frequency. Separating certain individuals seemed to affect the group differently, which indicates that the 'importance' of the animal to the group influences the group call rate. In this study, we have shown how social and environmental factors play a role in vocal communication in birds.
\end{abstract}

Key words: rooks, corvus frugilegus, contact calls, birds, communications

\section{Introduction}

Communication is all around us, we are all familiar with how to 'do it', and it is something that could not occur without social contact with others (McGregor 2005). But communication is not restricted to humans, all other animals also engage in various forms of communication, from vocal to tactile communication to using chemical cues. Over the last few decades the field of animal vocal communication has experienced a revolution in terms of methods and feasibility (Catchpole \& Slater 2008). Whereas before that, people had to draw spectrograms by hand, nowadays computers with

\footnotetext{
* University of Agricultural Sciences and Veterinary Medicine Cluj-Napoca, Faculty of Veterinary Medicine Cluj-Napoca, Discipline of Semiology, Ethology and Diagnostic Imaging

** Sub-Department of Animal Behaviour, Department of Zoology, University of Cambridge

*** Department of Experimental Psychology, University of Cambridge
}

Cluj Veterinary Journal, 15(1)/2009, pp. 33-43 
intricate software do all the hard work, enabling researchers to focus on more important aspects of communication rather than trying to improve the methods for studying it. With the programs available to us nowadays, we are certainly getting closer and closer to disentangling the various small pieces that make up a bout of communication, i.e. 'conversations' between two or more beings, and in a few cases even understand 'referential' communication, e.g. alarm calls in a situation of arousal when a predator is close by or begging calls produced by an animal that sees another carrying food and wants to gain some of it. Even so, we may never really know for sure what goes on in the mind of another animal, how other animals perceive the outside world (Nagel 1974), or even what they mean when they produce vocalizations, because of the fundamental differences between our minds' mechanisms (Quine 1973).

One of the various smaller fields in animal communication that people have started working on extensively is bird song (e.g. black-capped chickadees, Poecile atricapillus: Avey 2008; tyrant flycatchers, Tyrannidae: Hughes 2008; Eurasian jays, Garrulus glandarius: Goodwin 1949), although the vocal repertoire has also been explored in mammals like degus, Octodon degus (Long 2007). Generally, bird vocalizations are divided into songs - long, complex and spontaneous vocalizations that are commonly produced by males mainly during the breeding season - and calls - much shorter vocalizations that are produced by both sexes, throughout the year (Catchpole \& Slater 2008). The latter ones are given in certain contexts like danger, aggressive display or food desire and thus can be alarm calls, threat calls or begging calls.

The smallest structural units of bird songs and calls, named elements or notes, are in fact sounds that, combined in a certain way, form syllables, and syllables in turn are combined to make up phrases (Catchpole \& Slater 2008). A song may consist of a varying amount of the same or different phrases. A big advantage of vocal over non-vocal communication is that it uses sound as a means of communicating in situations where visibility is low or the distances are long (Catchpole \& Slater 2008). Sound is also a fast carrier of large amounts of information. Perhaps the energetic cost of sound production could be considered a disadvantage, but recent studies have shown that it is less expensive than other activities, such as flying (Oberweger \& Goller 2001, Ward et al. 2004). Having advantages that outweigh the cost, vocal communication thus seems to be the most suitable way of communicating for birds.

To pinpoint the meaning of vocalizations, one usually starts by combining observations of the bird's social relationship, interactions with its environment and recording calls and songs (Struhsaker 1967). This enables a better understanding of the context and thus of the meaning of a certain vocalization. However, grasping the whole range of utterances of one species is difficult to achieve, since there is a lot of variation, even between populations of the same species, stemming from different selective pressures and constraints working on their development, production, transmission and detection (Ryan \& Brenowitz 1985). A study with ravens (Corvus corax) suggests that vocalizations may very well vary even within populations, with new calls emerging and others being lost over time (EnggistDuebelin \& Pfister 2002). Therefore, one can only attempt to categorize as many different song or call types as possible, either by collecting observational data and sound recordings or by conducting experiments tailored to look at a certain vocalization and how it changes in different contexts.

Rooks are members of the Corvus genus, the biggest and most widespread of the Corvidae family. Consisting of 48 species, this genus also includes ravens, crows, jays, magpies, and jackdaws (Grzimek B. 2002). Observations show that the typical crows of the Corvus genus are characterized by a spectacular black plumage with metallic iridescence, food caching abilities, complex social 
interactions and hoarse voices, although still little remains known about their vocalizations. Hardy (1979) in a study on Black and Blue Jays (Cissilopha) hypothesized that social species produce a wider range of vocalizations than solitary-living species, a theory launched after studying and establishing the vocal repertoire of four closely related species of the Cyanocorax genus, in relation to their social habits. We would therefore expect to find a rich vocal repertoire in rooks (Corvus frugilegus), since it is a very social species, breeding and foraging close together in colonies and gathering at communal roosting sites (Røskaft \& Espmark 1982).

Using the approach described above and combining observations with recordings, in the present study we tried to determine the different call types making up our rooks' vocal repertoire. Further investigating the importance of one of the vocalizations, the contact call, we then conducted an experiment that looked at how the context can influence a vocalization.

\section{Materials and Methods}

\section{Subjects and housing}

In this study we used a group of eight rooks aged 6 years. The group consisted of five females and three males. For individual identification, they had been banded with coloured rings. All individuals were taken from the wild at a young age and hand-reared in captivity. The rooks were kept in a large outdoor aviary at the Sub-department of Animal Behaviour in Madingley, UK, measuring $10 \times 8 \times 4$ meters (length, width, height). The aviary consisted of two main compartments, similar in dimensions, separated from each other by a central small compartment.

\section{Procedure}

The present study consisted of two parts, each being designed to answer a different question: observations and an experiment. With the observations we wanted to establish the repertoire of rook vocalizations and determine the sex and individual differences in the calls; the experiment looked at the call frequency and call morphology differences between the test subject, its partner, and all other individuals. For both parts, a Canon MD 101 Digital Video CamRecorder and a Dell laptop with integrated SigmaTel stereo microphone array (SigmaTel High Definition Audio Codec) were used to make video and audio recordings.

\section{Observations}

We recorded vocalizations of all eight rooks over a period of 7 weeks (July-August 2008) for an average time of 1 to $2 \frac{1}{2}$ hours at different times of the day in order to avoid a possible bias caused by the time of day (e.g. influences of motivation or the nutritional status of the birds). The recordings' sample rate was of 44100 samples per second, with a resolution of 16 bits per sample and a stereo channel format. The recorded frequency ranged from 0 to $22000 \mathrm{~Hz}$. The audio files were stored in uncompressed wave format. After each vocalization notes regarding individual ID, time of vocalization, context of the vocalization, parallel behaviour accompanying the vocalization, distance to and visual contact with the partner and other members of the group and the subsequent vocalizations and parallel behaviour of the partner and other group members were taken. The vocalizations were classified into: neutral, agonistic (negative) and affiliative (positive).

\section{Experiment}

The experiment was conducted in July and August 2008. Each pair was only tested once a day, but in total received four test sessions: two sessions in two different conditions (see Fig. 1 and Fig. 2). The order in which the pairs were tested and the order in which the partners were isolated from the group on the other side of the aviary was pseudo randomized. 
Before each session, the birds were familiarized with the experimenter's presence. In both conditions, each session consisted of three phases, each of 10 minutes length: a pre-separation phase ((1) in Fig. 1 and 2), a separation phase ((2) in Fig. 1 and 2) and a post-separation phase, i.e. reunion of the partners in the group ((3) in Fig. 1 and 2).

During the pre-separation phase, both partners were in the group and could move around freely; at the beginning of the separation phase, experimenter 2 separated one or both partners from the group and at the end of the phase, the partners were reunited in the group for phase 3, a control phase. In condition 1, one of the individuals was physically, but not visually isolated from its partner and the rest of the group during phase 2 (Fig. 1).

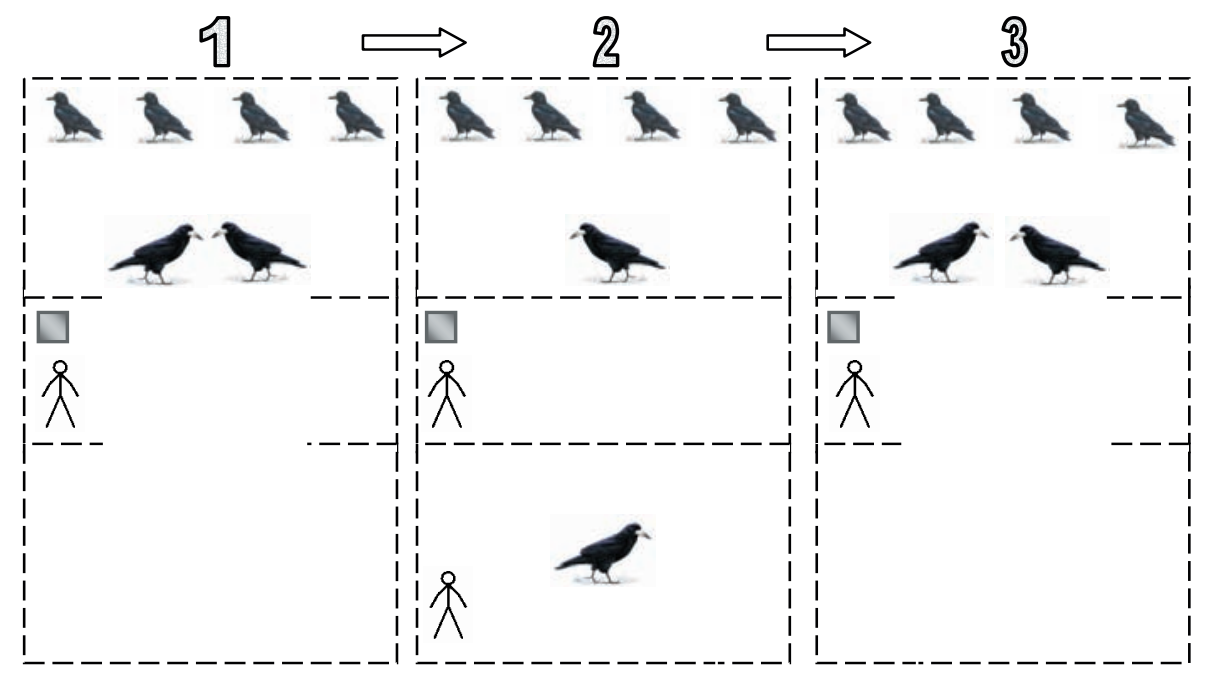

Fig. 1. Condition 1. Arrows show the three phases of the experiment: (1) pre-separation, (2) separation of the test subject (partner stays with group), (3) post-separation. The recording device is indicated by a square, experimenters are represented by figures. Dashed lines indicate wire mesh.

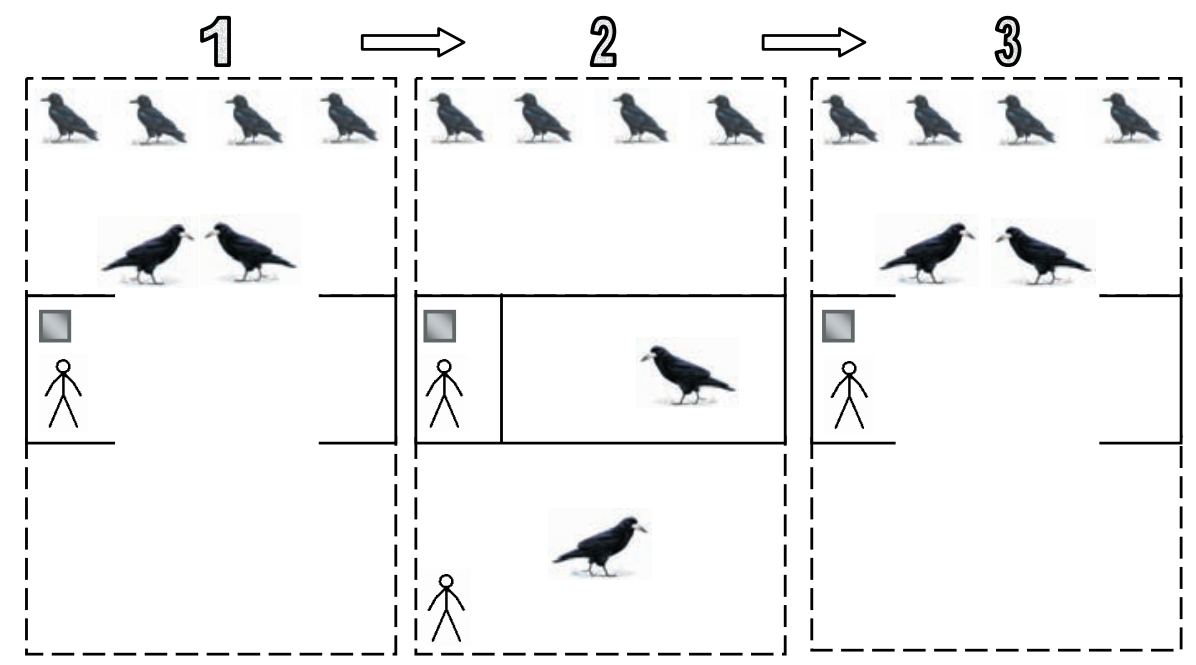

Fig. 2. Condition 2. The set up is the same as in condition 1 , with an exception during the separation phase (2), where both partners were separated from the group. Solid lines represent visually isolated compartments, dashed lines indicate wire mesh.

In condition 2, both partners were physically isolated from the group and each other. In addition, one was also visually isolated in the small compartment, while the other one could still see the group (Fig. 2). 
The latter condition was conducted to control for the influence of the group on the partner that stayed with the group in condition 1. In both conditions, observations of the group (including one of the partners in condition 1) were taken by experimenter 1 , whereas observations of the separated subject(s) during phase 2 were taken by experimenter 2 from outside the aviary.

\section{Data Analysis}

The dominance hierarchy was calculated with Matman on the basis of observed displacements (i.e. a bird retreats after having been approached by another). We used 10,000 randomizations and calculated the Landau's linearity index (h) and the directional consistency index to determine linearity of the hierarchy and the consistency of the result of the calculation (both indices lie between 1 and 0 ). An h of 1 indicates a linear hierarchy, whereas 0 point towards an ever-changing hierarchy.

For the analysis of the different call types, the vocalizations were extracted from the main recorded sound file, saved as wave files with the same format and added into a library, with the different call types categorized for each bird. Video data was then transferred onto the computer and analyzed together with data from the observation sheets. In order to save disk space and for easier storage the raw videos were then compressed using Microsoft Windows Movie Maker in windows media video file format with a $3 \mathrm{mb} / \mathrm{s}$ bit rate quality, preserving the original display size (720 x 480 pixels), aspect ratio (4:3), frames per second (30) and audio quality (16 bits per sample).

For recording, editing and analysis we used the following programs: Acoustica Premium (version 4.1.0, Acon Digital Media GmbH, 1994-2008), Avisoft-SASLab Pro (version 4.40, Avisoft Bioacoustics, 1990-2008), Windows Movie Maker (version 6.0, Microsoft Corporation); for statistical analysis we used SPSS 16.0 (SPSS Inc., 1989-2006). Using a spectrogram generated in Avisoft, we analyzed the following parameters: temporal parameters (duration of element, interval between elements, distance from start to maximum), sub-elements (number of elements), spectrum-based parameters (peak frequency, peak amplitude, fundamental frequency, minimum frequency, maximum frequency, frequencies of peaks, amplitude of peaks), locations of measurements (start of element, end of element, centre of element, maximum amplitude of element, mean spectrum of entire element, minimum parameter of entire element, maximum parameter of entire element). For classifying the vocalizations into different call types and looking at individual and sex differences, we used cluster analyses, Mann-Whitney U tests and Kruskal-Wallis tests. For analysing the number of contact calls given in the experiment, we calculated the average call number per individual across the two test sessions (separately for each condition) to rule out influences of the weather, the 'mood' of the birds, nutritional status etc. For analyses involving the 'other' six group members we did the same but also divided the number obtained by 6 (the total number of the birds excluding the test subject and the partner). We used Friedman ANOVAs and Wilcoxon matched pairs tests. All tests were nonparametric, twotailed, and alpha was set at 0.05 .

\section{Results}

\section{Dominance hierarchy}

The dominance hierarchy in the group was as follows: Plato (white ring) > Linnaeus (purple ring) > Mackintosh (red ring) $>$ Einstein (yellow ring) $>$ Darwin (green ring) $>$ Aristotle (red and blue rings) $>$ Da Vinci (blue ring) > Huxley (orange ring) $(\mathrm{h}=0.65$, directional consistency index $=0.98$ ). 


\section{Vocal repertoire}

We have established that the rook vocal repertoire consisted of nine structurally different calls, and several more variants. We have determined the function of three calls and have named them accordingly: contact and alarm calls, begging call, and submission calls. The remaining 6 calls and variants were named according to their acoustic similarity with other sounds: 'rattle', 'purr', 'click', 'hick-up', 'scream', and 'monkey' calls. Contact and alarm calls were the most common call vocalized by the group and were in fact the same call but used in different situations. Examples of some of the calls can be seen in figures 3, 4, 5, 6, 7, 8 .

Do the test subjects, their partners or the other birds vocalize more before, during or after the separation?

In both conditions, we found a significant difference between the average number of calls test subjects gave before, during and after the separation (Friedman ANOVA: condition 1: $\mathrm{n}=6, \mathrm{X}^{2}=6.09$, $\mathrm{p}=0.048$; condition $2: \mathrm{n}=6, \mathrm{X}^{2}=8.34, \mathrm{p}=0.016$; Fig.9). To determine the origin of the significant result, we conducted post-hoc Wilcoxon matched pairs tests. These revealed more calls of the test subject after than before the separation in both conditions (condition 1: $\mathrm{n}=6, \mathrm{Z}=2.20, \mathrm{p}=0.028$; condition 2: $n=6, Z=2.02, p=0.043$ ) and more calls after than during the separation in condition $2(n=6, Z=2.02, p=0.043)$.

Furthermore, a significant difference was found for the other group members in both conditions (Friedman ANOVA: condition 1: $\mathrm{n}=6, \mathrm{X}^{2}=7.60$, $\mathrm{p}=0.022$; condition $2: \mathrm{n}=6, \mathrm{X}^{2}=6.34, \mathrm{p}=0.042$; Fig.10). In both conditions, they produced more calls during the separation of an individual than before (Wilcoxon matched pairs tests: condition 1: $\mathrm{n}=6, \mathrm{Z}=2.20, \mathrm{p}=0.028$; condition 2 : $\mathrm{n}=6, \mathrm{Z}=1.99$, $\mathrm{p}=0.046$ ) and also more after than before the

Fig. 9. Box and whisker plots for the calls given by the test subjects in the three phases in condition 1. Boxes show the interquartile range; the box for the observers is divided by the median value. Whiskers indicate the largest and smallest value.
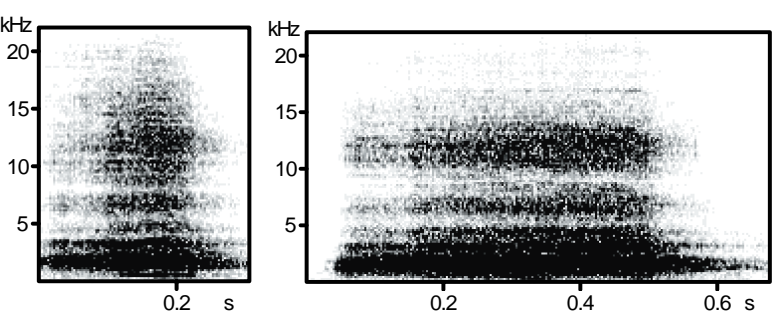

Fig. 3. Female contact calls
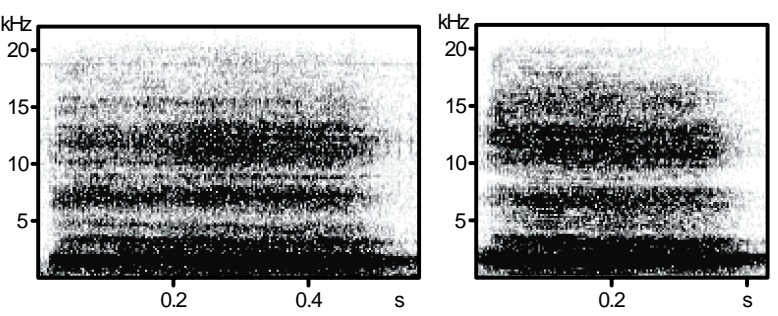

Fig. 4. Male contact calls

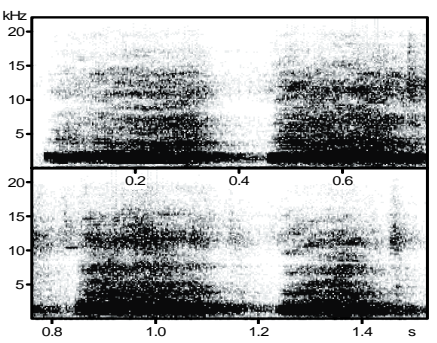

Fig. 5. Female begging call

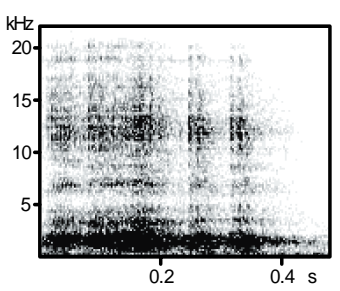

Fig. 6. Female 'rattle' call

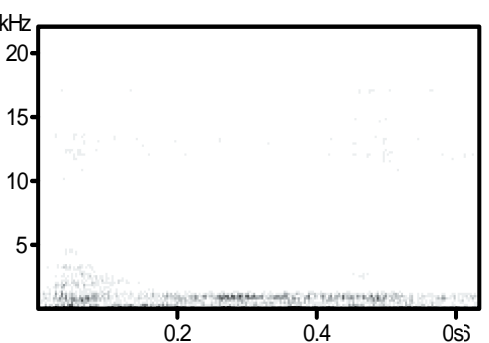

Fig. 7. Female 'hick-up' call

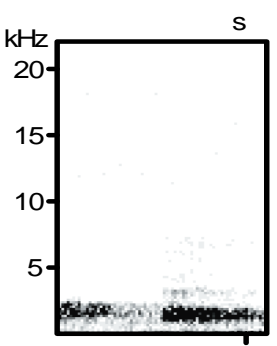

Fig. 8. Male 'click' call
Condition 1: Separ ated test subjects (on other side of the aviany)

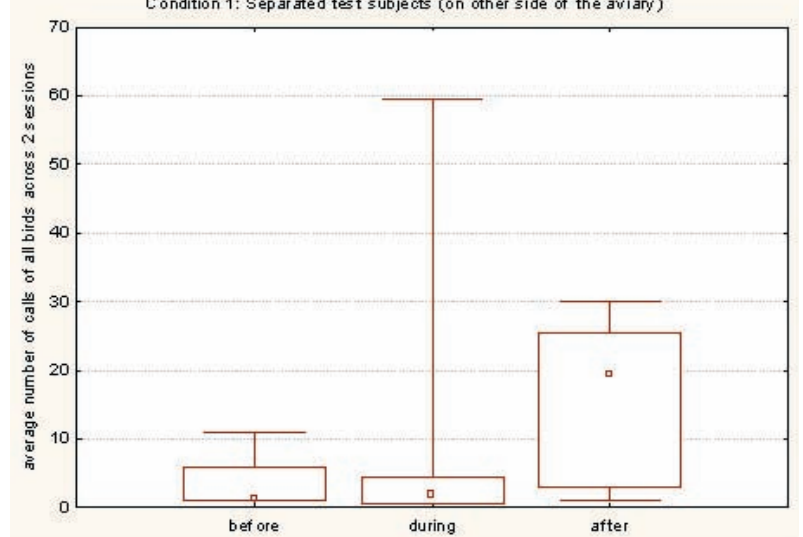


separation (condition 1: $\mathrm{n}=6, \mathrm{Z}=1.99, \mathrm{p}=0.046$; condition 2: $\mathrm{n}=6, \mathrm{Z}=2.20, \mathrm{p}=0.028$ ). There was no difference in the average number of calls the partner of the separated bird produced in any of the three phases in either of the two conditions.

Do the birds vocalize more before, during or after the separation of a certain individual?

Depending on which animal was separated, the birds called more or less before, during or after the separation in condition 1 . We found a significant difference between the average number of calls the birds produced before, during and after the separation when Da Vinci (Friedman ANOVA: $\left.\mathrm{n}=8, \mathrm{X}^{2}=8.96, \mathrm{p}=0.011\right)$, Darwin $\left(\mathrm{n}=8, \mathrm{X}^{2}=11.08\right.$, $\mathrm{p}=0.004)$, Mackintosh $\left(\mathrm{n}=8, \mathrm{X}^{2}=9.34, \mathrm{p}=0.009\right)$ and Plato $\left(n=8, X^{2}=6.34, p=0.042\right)$ were separated (e.g. Da Vinci, Fig.11). Results for the post-hoc Wilcoxon matched pairs tests can be found in Tab.1.

Similarly, we found significant differences in the number of calls in condition 2 when the following birds were separated: Da Vinci (Friedman ANOVA: $\left.n=8, X^{2}=9.34, p=0.009\right)$, Linnaeus $(n=8$, $\left.X^{2}=9.34, p=0.009\right)$, Mackintosh $\left(n=8, X^{2}=9.36\right.$, $\mathrm{p}=0.009)$, Aristotle $\left(\mathrm{n}=8, \mathrm{X}^{2}=7.36, \mathrm{p}=0.025\right)$, Plato $\left(\mathrm{n}=8, \mathrm{X}^{2}=9.36, \mathrm{p}=0.009\right)$ (e.g. Linnaeus, Fig. 12; for Wilcoxon matched pairs tests see Tab. 1).

Who vocalizes more: the subject, the partner, or the others?

We compared the average number of calls the test subjects, their partners and the other birds produced overall for each of the three phases (Friedman ANOVAS) and found no significant differences.

Is there a difference between the two conditions?

We looked at the three different phases separately and compared the average number of calls the test subjects, their partners and the other birds gave in condition 1 and 2. Except for the number of calls

Fig. 12. Box and whisker plots for the calls given during the separation of one bird in condition 2. Boxes show the interquartile range; the box for the observers is divided by the median value. Whiskers indicate the largest and smallest value.

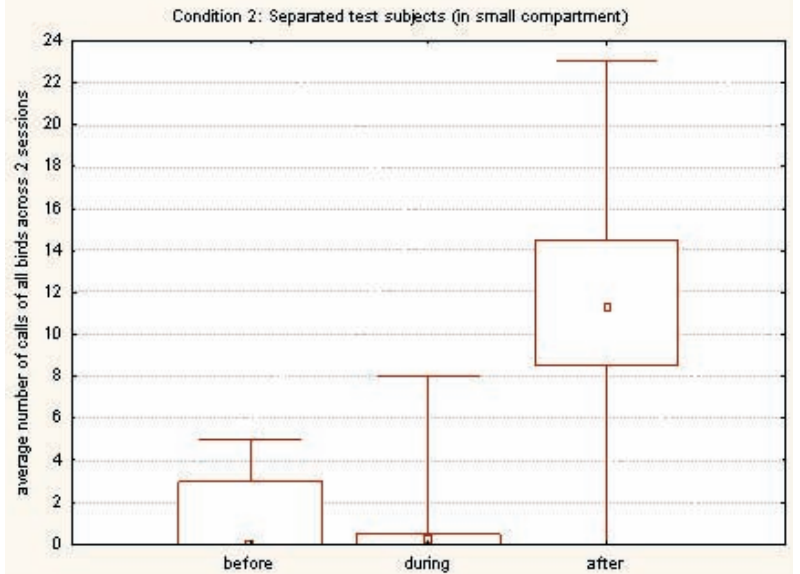

Fig. 10. Box and whisker plots for the calls given by the test subjects in the three phases in condition 2. Boxes show the interquartile range; the box for the observers is divided by the median value. Whiskers indicate the largest and smallest value.

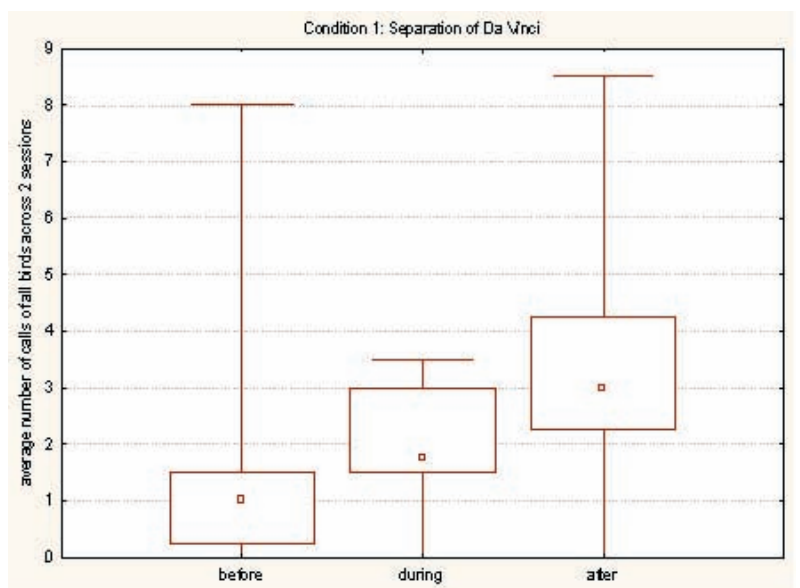

Fig. 11. Box and whisker plots for the calls given during the separation of one bird in condition 1 . Boxes show the interquartile range; the box for the observers is divided by the median value. Whiskers indicate the largest and smallest value.

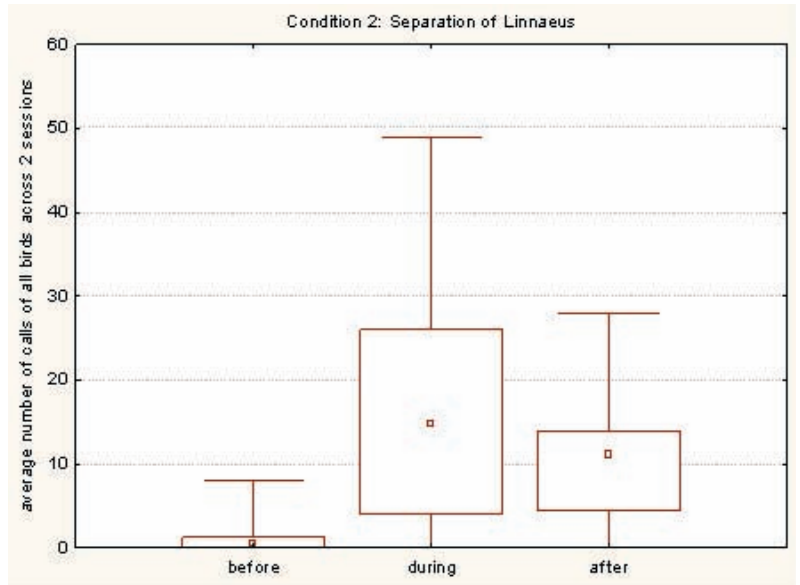


the subjects produced during the separation we found no significant differences. When separated, the subjects called more in condition 2 (in the small compartment) than in condition 1 (at the other side of the aviary) (Wilcoxon matched pairs test: $\mathrm{n}=6$, $\mathrm{Z}=2.02$, $\mathrm{p}=0.043$; Fig. 13).

Fig. 13. Box and whisker plots for the calls given by the test subjects in the two conditions. Boxes show the interquartile range; the box for the observers is divided by the median value. Whiskers indicate the largest and smallest value.

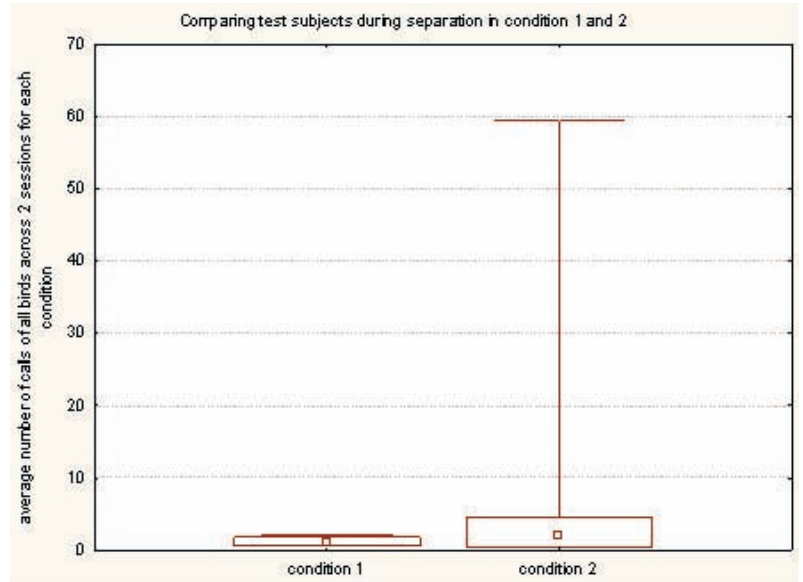

Tab. 1. Results for the comparison of the average number of calls all birds produced before, during and after the separation of certain individuals for (a) condition 1 and (b) condition 2.

\begin{tabular}{|l|l|c|c|l|}
\cline { 2 - 4 } \multicolumn{1}{c|}{ (a) } & phases compared & $\mathrm{Z}$ & $\mathrm{P}$ & \\
\hline \multirow{2}{*}{ Da Vinci } & during-after & 2.02 & 0.04 & more after \\
& after-before & 2.37 & 0.02 & more after \\
\hline \multirow{2}{*}{ Darwin } & before-during & 2.20 & 0.03 & more during \\
& during-after & 2.20 & 0.03 & more during \\
\hline \multirow{2}{*}{ Mackintosh } & before-during & 2.20 & 0.03 & more during \\
& after-before & 2.20 & 0.03 & more after \\
\hline \multirow{2}{*}{ Plato } & before-during & 1.99 & 0.05 & more during \\
& after-before & 2.20 & 0.03 & more after \\
\hline
\end{tabular}

\begin{tabular}{|l|l|c|c|l|}
\cline { 2 - 5 } \multicolumn{1}{c|}{$($ (b) } & phases compared & $\mathrm{Z}$ & $\mathrm{P}$ & \\
\hline \multirow{2}{*}{ Da Vinci } & before-during & 2.20 & 0.03 & more during \\
& after-before & 2.20 & 0.03 & more after \\
\hline Linnaeus & before-during & 2.20 & 0.03 & more during \\
& after-before & 2.20 & 0.03 & more after \\
\hline Mackintosh & before-during & 2.02 & 0.04 & more before \\
& during-after & 2.20 & 0.03 & more after \\
\hline Aristotle & after-before & 2.20 & 0.03 & more after \\
\hline \multirow{2}{*}{ Plato } & during-after & 2.02 & 0.04 & more after \\
& after-before & 2.20 & 0.03 & more after \\
\hline
\end{tabular}

\section{Discussion}

The present work revealed a large vocal repertoire in the studied group of rooks similar to the one Røskaft \& Espmark (1982) found in their group.

We found that the test subjects produced more contact calls after the separation from their partners than before it, irrespective of the condition, i.e. whether the separated partner was in the small compartment or in the other side of the aviary. When an individual was isolated from its partner in the small compartment, it gave more contact calls after than during the separation. Similar to postconflict affiliation, when rooks affiliate with each other after a fight in the group (Seed et al. 2007), rooks seem to call more after a stressful situation than before it. This points in the direction of stress as an important influence of calling for the subjects. Thus, due spending energy on increasing their heart 
and breathing rate, they might lack the energy for calling during separation. Also, the restricted dark space may be an unnatural situation they want to get out of by any means, and for them, the best way for doing that may seem to be through the cracks, where they can see light coming in. Continuously trying every possible way of escaping, calling becomes less of a priority, and as stated above, they could at the same time save energy for the flight. The separated bird produced fewer calls in the small compartment in condition 2, in which he could not see the partner, than afterwards, when he was reunited with his partner. Nevertheless, compared to condition 1, the subjects produced more contact calls during separation in condition 2, indicating an overall higher level of arousal in condition 2, which may be due to the additional stress of lack of visual contact with the separated bird. This has also been a noted result in a study on marmosets, where the subjects produced a "separation phee" call during short periods of absence from the group (Norcross et al. 1999). A study that is currently being conducted with chimpanzees looks at the influence of visibility in the forest on call rate and may reveal similar results (Slocombe et al. in prep.).

Also, the other group members gave more contact calls during the separation of a bird than before, and more after than during the separation, further supporting the idea of stress as a factor.

Interestingly, the partner of the isolated bird did not change the rate of contact calls in any of the phases in either of the conditions. In both conditions the partner was not physically constricted, it could see the group members and could freely communicate with them. Only in the second phase of the second condition the partner was physically isolated but in a familiar enclosure comparable in size and visibility with the one it was separated from. So, one explanation can be that the partner could better assess the situation than the test subject and thus would communicate at a regular frequency. Another explanation could be that the distance and physical isolation from the group make the bird instinctively try to conceal its location to an eventual predator, and so minimize the chances of being in imminent danger. Instead of using compromising vocalizations it spends its energy on finding a way to get to its partner or the group members. This is because for a social species, rooks are safer when gathered in large groups, while one bird is more vulnerable on its own.

Separating certain individuals seemed to affect the other group members differently, indicating an influence of the 'importance' of the animal to the group on the call rate, potentially arising from the dominance status or the sex of the given bird. Social dominance hierarchy has been shown to play an important role in determining the long-distance call frequency and structure in male chimpanzees (Pan troglodytes), in the way that high-ranking males produce more calls than low-ranking ones (Mitani and Nishida 1993).

Other studies have shown that not necessarily the rate, but for example the structure of vocalizations changes when animals are separated from their group or partner. The oldest example in this direction is the 'sympathetic song' of two Australian magpies (Gymnorhina tibicen). The two birds had succeeded in learning a flute song that both had been exposed to and managed to reproduce it as a duet, in which each one uttered a half while the other one would finish the song (Waite 1903). Gwinner and Kneutgen have shown that because both the common raven (Corvus corax) and the common shama (Copsychus malabaricus) bond for life and have individually different vocalizations they have a much stronger vocal relationship. When an individual had not been in the presence of its mate for some time, the latter one would produce the missing partner's vocalizations. Upon hearing its own vocalizations, the mate would then return to the caller (Gwinner and Kneutgen 1962). The same phenomenon has also been reported for the eastern bluebird (Sialia sialis) (Morton et al. 1978) and the black-headed grosbeak (Pheucticus melanocephalus) (Ritchison 1983). 
Similar findings have been reported in nonhuman primates that show fine differences in their call structure as a result of social changes. When paired with a new mate, pigmy marmosets (Cebuella pygmaea) modify their trill vocalizations structure (Snowdown and Elowson 1999). Periods of social instability determine the emergence of novel variants and transformations in frequency structure of the 'combined harmonic' calls in female Campbell's monkeys (Cercopithecus campbelli) (LeMasson and Hausberger 2004). An acoustic variability has been demonstrated to occur in the male chimpanzee (Pan troglodytes) typical long-distance calls, the pant hoot, in relation to pre and post-traveling situations of temporary associated parties (Mitani and Brandt 1994).

Future studies could take into account the stress of the act of separating a bird on the group and include control experiments to rule out the actual separating as an influence on the call rate, e.g. by catching a bird, letting it go again and measuring the contact call rate immediately afterwards. Furthermore, to increase the sample size of just three rook pairs, more data should be taken from different rook groups. In addition, field observations could prove helpful in determining the actual relevance of our study, by noting the different stressful factors that can naturally occur (e.g. predator chasing one of the partners, or partner dies). Recording quality could be improved by installing microphones within the aviary or the rook territory to reduce the stress factor created by the presence of an experimenter. This would generate more accurate data, as the recordings could continue over a longer period of time. Miniature microphones could also be installed directly on the individuals' bodies to reduce the possibility of confounding certain birds' vocalizations. In order to better determine the context in which one vocalization is being produced, CCTV cameras could be installed in different angles within the aviary, thus reducing the necessity of human presence and in this way eliminating the additional stress created otherwise.

Overall, we have shown that vocal communication in rooks, as a highly social species, is a very flexible process influenced by different social and environmental factors.

\section{References}

1. Avey M. T., Quince A. F. \& Sturdy C. B. 2008. Seasonal and diurnal patterns of black-capped chickadee (Poecile atricapillus) vocal production. Behavioural Processes, 77, 149-155.

2. Catchpole, C. K. \& Slater P. J. B. 2008. Bird song. Biological themes and variations. Cambridge: Cambridge University Press.

3. Enggist-Duebelin, P. \& Pfister, U. 2002. Cultural transmission of vocalizations in ravens, Corvus corax. Animal Behaviour, 64, 831-841.

4. Goodwin D. 1949. Notes on voice and display of the jay. British Birds, 42, 278-287

5. Grzimek B. 2002. Crows and Jays. In: Grzimek's Animal Life Encyclopedia, 2nd edition, vol. 11, Birds $I V$, pp. 503-511. (Ed by M. Hutchins, J. A. Jackson, W. J. Bock \& D. Olendorf) Farmington Hills, MI: Gale Group.

6. Gwinner, E. \& Kneutgen, J., 1962. Über die biologische Bedeutung der "zweckdienlichen" Anwendung erlernter Laute bei Vögeln. Zeitschrift fur Tierpsychologie, 19, 692-696

7. Hardy, J. W. 1979. Vocal repertoire and its possible evolution in the Black and Blue Jays (Cissilopha). The Wilson Bulletin, 91, 187-201.

8. Hughes A. L. 2008. Temporal pattern of vocalization type usage in singing sessions of male tyrant flycatchers (Tyrannidae). Journal of Avian Biology, 39, 24-29.

9. Lemasson, A. \& Hausberger, M., 2004. Patterns of vocal sharing and social dynamics in a captive group of Campbell's monkeys (Cercopithecus campbelli). Journal of Comparative Psychology, 118, 347-359.

10.Long, C. V. 2007. Vocalisations of the degu Octodon degus, a social caviomorph rodent. Bioacoustics, $16,223-244$.

11.Mitani, J.C. and J. Gros-Louis., 1998. Chorusing and call convergence in chimpanzees: Tests of three hypotheses. Behaviour 135. 1041-1064. 
12.Mitani, J. C. \& Brandt, K. L. 1994. Social factors influence the acoustic variability in the long-distance calls of male chimpanzees. Ethology, 96, 233-252.

13. Mitani, J.C. and T. Nishida. 1993. Contexts and social correlates of long distance calling by male chimpanzees. Animal Behaviour 45, 735-746.

14.McGregor, P. K. 2005. Introduction. In: Animal communication networks. pp. ix-xi. (Ed. by P. McGregor) Cambridge: Cambridge University Press.

15.Morton, E.S., Geitgey, M. S. \& McGrath, S., 1978. Responses to apparent female adultery. American Naturalist 112, 968-971

16.Nagel, T. 1974. What Is It Like to Be a Bat? The Philosophical Review, 83, 435-450.

17.Norcross J.L., Newman J.D., Cofrancesco L.M. 1999. Context and sex differences exist in the acoustic structure of phee calls by newly-paired common marmosets (Callithrix jacchus). American Journal of Primatology, 49, 165-181.

18. Oberweger, K. \& Goller, F. 2001. The metabolic cost of birdsong production. Journal of Experimental Biology, 204, 3379-3388.

19. Quine, W. V. 1973. On the reasons for the indeterminacy of translation. Journal of Philosophy, 12, 178183.

20.Ritchison, G., 1983: Possible "deceptive" use of song by female black-headed grosbeaks. Condor 85, 250-251

21.Ryan, M.J. \& Brenowitz, A.E. 1985. The Role of Body Size, Phylogeny, and Ambient Noise in the Evolution of Bird Song. The American Naturalist, 126, 87.

22.Seed, A., Clayton, N. S. \& Emery, N. J. 2007. Postconflict third-party affiliation in rooks, Corvus frugilegus. Current Biology, 17, 157-158.

23.Snowdon, C. T. \& Elowson, A. M., 1999. Pygmy marmosets modify call structure when paired. Ethology, 105, 893-908.

24.Struhsaker T. T. 1967. Behavior of Vervet Monkeys and other Cercopithecines, Science, 156, 1197.

25. Ward S., Lampe H. M. \& Slater P. J. B. 2004. Singing is not energetically demanding for pied flycatchers, Ficedula hypoleuca. Behavioural Ecology, 15, 477-484.

26. Waite, E.R., 1903. Sympathetic song in birds. Nature 68, 322 